\title{
The Semantic Network as a Promising Information Platform in the Mining Industry
}

\author{
Gulnur Alkhanova ${ }^{1}$, Dmitry Stenin ${ }^{2,}$, and Serik Zhuzbaev ${ }^{3}$ \\ ${ }^{1}$ Kazakh Humanitarian-Law Innovative University, Semey, Kazakhstan \\ ${ }^{2}$ T.F. Gorbachev Kuzbass State Technical University, 650000, Kemerovo, Russian Federation \\ ${ }^{3}$ L.N. Gumilyov Eurasian University, Nur - Sultan, Kazakhstan
}

\begin{abstract}
The issues of digitalization of the mining industry, including open-pit mining, are considered. The issues of the use of semantic systems as an element of information flows, allowing to determine and select the right decisions in the process of mining management, in particular, in the mining of minerals by open mining, are covered. The article formulates requirements for semantic networks for the description of material in computer-aided learning tools and analyzes the most well-known models of semantic networks.
\end{abstract}

\section{Introduction}

Open pit mining is a complex technological process consisting of many operations. At each stage of this process, due to different mining and mining and geological conditions, the solution of any issues may be different. In order to eliminate the human factor and, possibly, the wrong decision, it is advisable to use information systems and technologies as an element of the digitalization of the mining industry and, in particular, semantic models and networks. Semantic networks are, first of all, a capable mathematical model that gives an idea of knowledge around the subject area (software), one of the most important areas of insights produced. In the scientific literature depicts numerous elective representations of models of semantic networks. They are designed to highlight the range of orders in several programs.

In general, semantic ordering can be represented as an expression:

$$
\mathrm{S}=\left(\mathrm{O}, \mathrm{R}_{1}, \mathrm{R}_{2}, \ldots, \mathrm{R}_{\mathrm{k}}\right)
$$

where $O$ - objects of division in a certain interval of a subject;

$R_{i}=1, \mathrm{n}$ - numerous connections between objects;

$i$ - type of relationship.

Web-based applications are widely used, which inevitably leads to the need to create appropriate mechanisms for the paradigm of web services that can provide stable and reliable execution flows [1].

The promotion of the model $\mathrm{S}$ begins with a specification of the needs for it with a computer program under the thought. In our case, a computer program is a demonstration

\footnotetext{
* Corresponding author: $\underline{\text { sdv.ea@ } @ \text { kuzstu.ru }}$
} 
of an informational presentation about instructional material, on the one hand, and a demonstration of information about a doubler, on the other. In this case, the most necessary is what is needed.

1. The strategy of introducing initial data should reflect all the conditions of the structure of assignments, mining facilities, their properties and relationships between facilities.

2. The presentation strategy should allow ignoring unimportant properties of objects.

3. The ability to image within the dialect of demonstration standards.

4. The plausibility of the image dialect models of programs specific training courses.

5. The ability to depict (construct, create) a dialect model of a person in an educational program.

6. The introduction strategy should reflect all the conditions of the structure of assignments, objects, their properties and relations between objects.

7. The strategy of introduction should allow you to neglect the insignificant properties of objects, and in general.

8. The ability to image within the dialect demonstration of state educational standards.

9. The credibility of the image dialect models of programs specific training courses.

10. The ability to depict (construct, create) dialectal show of individual programs.

11. Reliability of accounting (reflection and presentation) in the framework of manifestations of individual psychological and pedagogical qualities (status) of doublers.

12. The validity of taking into account in the framework of the models of individual moments of instructive training (the introduction of the most instructive tissue, the combination of the tissue in question, redundancy of traversal, distinctive types and levels of control, self-control of the understudy, etc.).

13.Multi-level organization of multiple operations with the model.

$$
\mathrm{L}=\left(\mathrm{L}_{1}, \mathrm{~L}_{2}, \mathrm{~L}_{3}, \ldots\right)
$$

The levels of operations in (2) we are going to specify below, and here we proceed to the creation of preconditions for the model created.

14. Ability to work with locally deficient, locally repetitive and locally conflicting data. Therefore:

- the availability of operations to confirm, localize and identify inconsistencies;

- the quality of the "work" model should depend on its completeness.

15. Graphic explanation of the fundamental operations on semantic ordering. This premise is due to the receptivity and extraordinary comfort for clients (methodologists, trainers, trained) of the schedule for clarifying the semantic organization and operations on it. Now back to expression (2), where:

$\mathrm{L}_{1}$ is the level of interface operations. The following operations are possible at this level:

$\mathrm{L}_{1,1}$ - creation (re-creation or layout) of any component of the model;

$\mathrm{L}_{1,2}$ - change of any existing component of the model;

$\mathrm{L}_{1,3}$ - exclusion (by accident or permanently) of any component of the demonstration

$\mathrm{L}_{1,4}$ - designation of any component of the model;

$\mathrm{L}_{1,5}$ - detection (visualization) of any part of the show, etc.

$\mathrm{L}_{2}-$ level of basic operations from the demonstration. Take after the premise to this level of operations.

1. Working with a single set of data structures for the selected categorical database of information is practically software.

2. Work with information at various levels of detail of their implementation. This premise legitimizes the representation of operations of conglomeration (composition) and their semantic translation in the form of segments, subsections, personalized lessons, addresses, and other organizational units of the instructional descriptor. 
3. In addition to accumulation operations, it is important to recognize operations. Logical completion in the sense of a formal and scientific justification. This precondition is based on the completeness of the set-theoretic operations of the components shown.

$\mathrm{L}_{3}$ - the level of operations confirm and determine the fight.

Some sources of information in a computer program are: mechanized sources (scanners and OCR systems, databases and information) or specialists.

Since we most often bargain with a different set of sources, the input information flow P1 is recognized by: nearby deficit, irregularity, neighborhood with excess (Fig. 1)

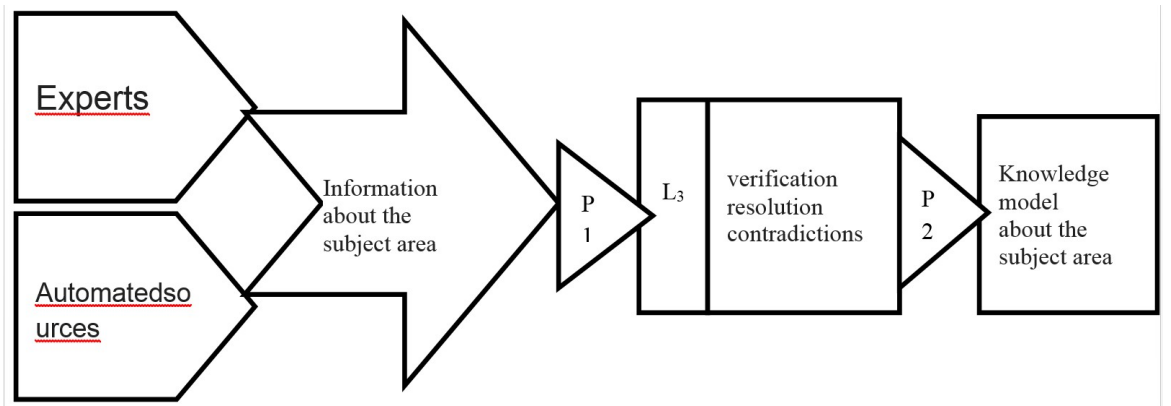

Fig. 1. Information flow diagramю

After applying the operations of the lesson L3 to the flow P1, the flow P2 is stable, not redundant and complete.

$\mathrm{L}_{4}-$ operation on the functioning of the show semantic systems, as demonstrated

multigraph.

$\mathrm{L}_{5}$ - the level of the programmed data retrieval and update operations.

$\mathrm{L}_{6}$ is the level of administration procedures for the entire set of operations.

\section{Discussion}

Semantic systems are informational graphics that include hubs and connections (circular segments or bolts) between hubs. Hubs speak to objects or concepts, and connections to connections between hubs. Thus, a semantic layout can be a coordinated diagram. When printing, the hubs are regularly processed in circles or areas, and the connections are drawn in the form of bolts between the circles. Primary computer use of semantic systems for artificial understanding and machine interpretation, but the preceding forms have long been used in reasoning, psychology and linguistics. The monstrous Worldwide semantic ranking map can be an expansive semantic network. Common to all semantic systems is an explanatory graphical representation that can be used to transfer information and strengthen mechanized thinking structures that are close to information. Some forms are purely random, but others are formally characterized by a framework of justification. Acceptance after - these are the six most common types of semantic systems:

Network definitions emphasize a subtype or link between sorting concepts and the recently characterized subtype. Since definitions are adjusted by definition, the data in these systems is expected to be mostly correct.

The claims organization is intended to approve recommendations. Not at all like systems, definitions, data within the framework of explanations of an organization are considered conditionally correct if they do not have an unambiguous stamp of a modular administrator.

Implication systems use sentence as an essential relationship for interacting epicenters of activity. They can use to speak with constructs of belief, causation, or reasoning. 
Executable systems include several components, such as transferring tokens or related strategies that can draw conclusions, send messages, or search for projects and associations.

Training systems build or expand their views, getting information from the cases. Modern information can change the ancient organization by including and evacuating concentrators and circular segments or by changing the number.

Hybrid systems combine two or more past strategies, either in the same organization, or in isolated, but closely associated systems [3-4].

Some semantic systems were specifically planned to update theories around a person's cognitive tools, while others were created mainly for computer efficiency. In some cases, computational problems can lead to the same conclusions as mental evidence. The contrast between definitions and for illustration, overconfident systems have a close parallel with the contrast between semantic memory and long wave memory [5].

Computerized semantic systems are the representation of a set of concepts or the definition of the fundamental organization of concepts in training. Semantic systems require the study of the basic interaction between the conscious substances of students. When building semantic systems, doublers should be able to analyze the structure of their information, which can help them integrate modern information into existing information structures. The result is a convincing use of the information received [6-8].

Improving semantic systems requires:

- reorganization of education;

- clear representation of concepts and associations between them;

- this will allow you to keep in mind and collect information, as well as increase the ability to apply information in unused conditions;

- combining modern concepts with modern concepts and ideas that promote understanding.

\section{Results}

The most important areas of research: the organization of research. It turned out that the creation of semantic networks and cognitive maps can be a consequence of the exact mapping of the structure of information. The organization of these semantic systems will help illustrate their cognitive structures.

Among the models of semantic systems created in the following decades, the most popular are: extended semantic systems, heterogeneous semantic systems, a generalized presentation of information in almost the subject area.

Four fundamental standards in model development.

1. The open character of numerous subject ranges, the consistent development in them of performing tasks. This requires enhanced functionality to show in the box. It is emphasized that it is reasonable to do this not at the expense of time-consuming programming, but at the expense of introducing into the demonstration unused data of revelation in a form convenient for a person. Therefore, they must be replaced by an internal representation.

2. The dialect of information representation should be focused on independent intra-system training. Thus, the implied display of information must be basic, of the same kind, consistent with the measure of homogeneity, and must have a basic premise. Semantic networks best meet these criteria.

3. They reveal the open character of numerous subject areas, the consistent development of the length of tasks. This requires an enhanced demonstration utility under a suitable heading. It is emphasized that it is advisable to carry out not due to time-consuming programming, but due to the introduction of modern data of revelation into a form convenient for a person. Therefore, they must be replaced by an internal representation. 
4. The dialect of information representation should be focused on independent intra-system training. Therefore, the presentation of information should be simple, of the same type, should be consistent with the principle of homogeneity and have a basic premise. Semantic systems best meet these criteria.

5. The open nature of numerous subject areas, the consistent expansion of the range of tasks were unraveled in them. This requires enhancing the usefulness of the show in a suitable course. It is emphasized that it is advisable to carry out this not due to time-consuming programming, but by entering modern explanatory data into the show in a human-friendly frame. Therefore, they must be replaced by an internal representation.

6. The dialect of the presentation of information should be focused on independent intrasystem processing. Therefore, the presentation of information should be simple, of the same type, consistent with the homogeneity model and have a basic premise. Semantic systems best meet these criteria.

7. There may be a need for coherent strategies for addressing the issues of input and processing information that are different from the rationale of predicates, not enough to justify the etymological component, the organization of the interface, etc.

8. The demonstration should be supported by suitable devices aimed at expanding the classes of application frameworks and output clients.

In the understanding of these standards, the device of enhanced semantic systems is proposed. To get rid of the heterogeneity of generally accepted semantic systems arising from the proximity of quantifiers, and from the set of objects connected by connections, connections connected by connections, etc., are presented: vertices that are compared with the names of relations and vertices when performing the "decoupling" of components. A vertex of a circular segment breaks down when one edge is arranged and interacts with the beat-relative, and the other edge with vertices of objects. To efficiently organize large volumes of information and code, the structure implementing the demonstration of the NOSS should be divided into modules. The question shows what classes, objects, and object occurrences say. The course contains reachable rules and images of computable and non-computable properties. Properties and strategies have accessibility property from other classes and modules.

The result of the conclusion is characterized by the degree of honesty of the conclusion, the degree of certainty within the framework of the decisive and necessarily evaluated on the basis of the primary two-component truth of the conclusion. Due to the drawbacks of the well-known fluffy induction procedure, NOSS uses a direct exit procedure with subtraction, which allows the program to be viewed from an explanatory point of view as performing fluffy input, and from a procedural point as a call to recursive methods or functions. The NOSS uses an object-oriented approach. Subsequently, each show launch program takes place in an arrangement lesson and is considered as a strategy in this course. Rules are divided into built-in and user-defined rules.

The FSNL dialect (Fluffy Semantic Organize Dialect) was created to display information and organize code. FSNL is an object-oriented dialect without strict writing, designed to work with semantic systems and has the properties necessary to form a model. The FSNL dialect can be used both to understand the problems of fake ideas and to understand common programming problems. Object-oriented dynamic networks for fuzzy cases that allow you to represent knowledge about objects and classes of objects that are fuzzy in nature, as well as to model their changes in time. Within the framework of the described approach, a mechanism is proposed that allows obtaining new knowledge based on basic knowledge and differs significantly from the known methods used in existing knowledge representation models.

In addition to the above, regarding objects and their properties, we draw attention to the following important point: the properties of the object and the object itself are closely 
interrelated and cannot exist separately. Properties themselves do not exist without an object, since some object is their manifestation, and properties cannot be seen, understood, or even described without it [9-10].

\section{Conclusion}

1. Prerequisites for the numerical device for constructing any module in the information system of any mining enterprise, most of which are: the ability to display specific plans and prepare courses within the dialect of showing standards; - the ability to reflect within the framework of the demonstration the state of the processes and stages of production. It turns out that semantic systems most satisfy these needs.

2. A review of most models of semantic systems: enhanced semantic systems, heterogeneous semantic systems, fluffy semantic systems, and a generalized knowledge representation around the subject area.

3. It turned out that the analyzed models do not fully take into account the main points and needs of the mining industry. Subsequently, the task of changing the demonstration of semantic location under these needs.

\section{References}

1. R. Vaculın, K. Wiesner, K. Sycara, Modelling (IEEE Computer Society, Los Alamitos, 2008)

2. V.I. Esin, S.G. Rassomakhin, V.M. Grachev, N.G. Polukhina, bulletin of the Lebedev Physics Institute, 41:5, 123 (2014)

3. A. Fedorchenko, A. Chechulin, I. Kotenko, PDP, 23, 559 (2015)

4. A.I.H. Suhaimi, Y. Goto, J. Cheng, Transactions on information and systems, E97:6, 1516 (2014)

5. H.Y. Zhao, X.Y. Liu, Z. Jing, Applied mechanics and materials, 397:400, 2536 (2013)

6. N.V. Mostovaya, E.V. Lebedenko, Proceed. XXIV Int. Op. Sci. Conf., 97, 200 (2019)

7. O. Outhavong, Security in a distributed object-oriented database in the context of a three-tier architecture illustrated with the implementation of a flight safety information system (CKUF, Beijing, 2002)

8. A. Lehew, Globalization of the enterprise database environment: a qualitative study of data security experience (SMJS, Praha, 2005)

9. I.I. Artic, Cybernetics and Systems Analysis, 52(1), 38 (2016)

10. D. A. Terletskyi1, A. I. Provota, I. Arch., 1601, 01635 (2016) 